\title{
USING TEXT MINING AND RANDOM FORESTS FOR AUTHOR IDENTIFICATION. THE CASE OF CLARIVATE WEB OF SCIENCE DATABASE
}

\author{
Marin FOTACHE \\ Alexandru Ioan Cuza University of Iasi, Romania \\ fotache@uaic.ro
}

\begin{abstract}
Author identification/disambiguation is a major topic of interest not only for scientific databases and various information providers, but also for universities and research institutes in reporting scientific production. This paper presents the module implemented at Alexandru Ioan Cuza University of Iasi for identifying the papers indexed in Clarivate (ISI) Web of Science database whose (co)authors are UAIC members. Based on main metadata available (in various extents and rigor) for Clarivate indexed papers and information gathered from some of the UAIC people (e-mail addresses, ORCID, ResearcherID), papers with highplausible and also high-implausible authorship are first extracted. Titles of these papers are subject to basic text processing methods in building frequency tables and other metrics of unigrams and bigrams for people, domains of research and departments. Computed metrics are used as variables in random forest classification models for predicting the authorship for UAIC members in the case of papers lacking rigorous metadata. Model performance and variable importance are presented and discussed.
\end{abstract}

Keywords: Author identification, ISI Web of science, text mining, random forests.

JEL classification: C38, D83, L86

DOI: $10.12948 / \mathrm{ie} 2019.01 .01$

\section{Introduction. Problem statement}

Reporting scientific production is one common annoyance for Romanian universities since identical information is required repeatedly, in various formats and degrees of details. Apparently, almost all Romanian agencies for higher education funding and assessment suffer the Alzheimer's Disease, since information is always lost from year to year, and every once in a while, a new report is required for the last one/three/five years, accompanied by various impact and influence scores.

The most affected are researchers in areas with large volumes of published papers, such as physics and chemistry. Copying into reporting worksheets the DOI's, wos codes, titles, authors, issns, isbns, journal and conference names, volumes, issues, etc. of tens and sometimes hundreds of papers may keep many researchers away from writing other papers (admittedly, this can bring some comfort to their non-research fellows and in some extreme cases can even be beneficial for the advancement of science). Not to mention the inconsistencies occurring when multiple authors report a common paper. Removing duplicate papers at department, faculty and university level can prove a daunting task.

In 2015 Al. I. Cuza University of Iasi (UAIC) started funding a project for scanning the major research databases and extract relevant data about papers whose authors are, or seem to be, UAIC members. Currently the implemented software platform displays to each faculty member and researcher a list of works whose presumable (co)author she/he is, and the person confirms of reject the authorship. 
The problem of author identification or disambiguation has been of great interest for digital libraries, research databases and other types of information providers. In Fereira et al.'s taxonomy [1] the author assignment is based mainly on classification and clustering. Zantout et. al.'s approach [2] is to extract chunk of text from documents with uncertain authorship and to compare the chunks with some text known to be written by the would-be author; the degree overlapping would be a good predictor of authorship, no matter the language of the text. Other approaches include, but not limited to, stylometry [3] and topic modeling [4].

This paper presents the solution implemented at UAIC for author identification for papers indexed in Clarivate (ISI) Web of Science. Data are collected from Clarivate and UAIC databases (section 2). Section 3 describes how the dataset is created using basic text mining techniques. Predictive models (classification) are built with Random Forests and assessed for performance. Section 4 provides some insights about the results.

\section{Clarivate ISI Web of Science metadata and UAIC members data}

Clarivate (ISI) Web of Science is the main reference for Romanian higher education agencies in assessing research production. The database provides rich metadata about papers and journals. Table 1 presents the main markers relevant for building our models.

Table 1. ISI Web of Science markers used in the models

\begin{tabular}{|l|l|l|}
\hline \multicolumn{1}{|c|}{ Marker(s) } & \multicolumn{1}{|c|}{ Description } & \multicolumn{1}{|c|}{ Comments } \\
\hline UT & ISI Unique Article Identifier & Paper identifier; ex: `WOS:000418463600052` \\
\hline AF & Authors (of the article) Full Name & $\begin{array}{l}\text { Ex: `Fotache, Marin; Hrubaru, I`; main problem: } \\
\text { not always available }\end{array}$ \\
\hline AU & Article Authors & $\begin{array}{l}\text { Ex: `Fotache, M., Hrubaru, I.'; available and } \\
\text { reliable }\end{array}$ \\
\hline BF, BA & Book authors & Similar to AF and BA, but for books \\
\hline EM & E-mail address & $\begin{array}{l}\text { Usually available only for the first author; } \\
\text { frequently missing }\end{array}$ \\
\hline OI & $\begin{array}{l}\text { ORCID Identifier (Open } \\
\text { Researcher and Contributor ID) }\end{array}$ & $\begin{array}{l}\text { Missing frequently or available only for a subset of } \\
\text { the authors; }\end{array}$ \\
\hline RI & ResearcherID Number & $\begin{array}{l}\text { Missing frequently or available only for a subset of } \\
\text { the authors; }\end{array}$ \\
\hline C1 & Author Address & $\begin{array}{l}\text { Author affiliation; missing frequently or available } \\
\text { only for a subset of the authors; }\end{array}$ \\
\hline TI & Document Title & Article/book title \\
\hline
\end{tabular}

Author identification is simple when markers like e-mail, ORCID and ResearchedID are available, since they are unique for a person. Unfortunately, this is not always the case - as in many cases these data are missing - see the left side of figure 1.

From UAIC database the main collected data were:

- family name, surname;

- author's main domain of interest and the department where she/he is affiliated;

- various aliases (useful especially for the people that changed their family names, e.g. when getting married);

- declared e-mails, ORCID, and ResearchedID.

The chart on the right side of figure 1 shows that a large proportion of UAIC members did not fill in their ORCID and ResearchedID. 

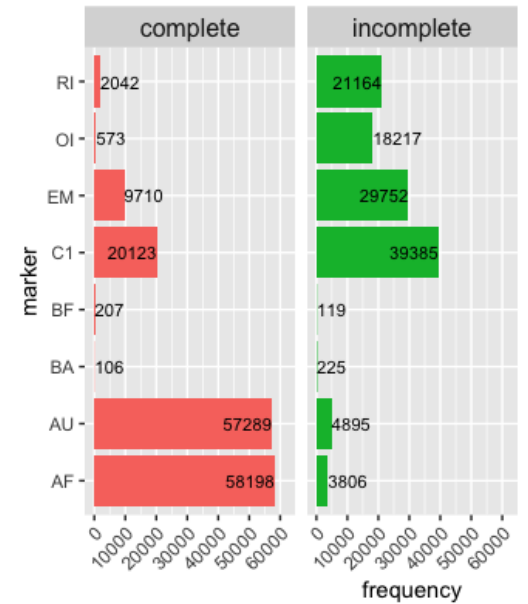

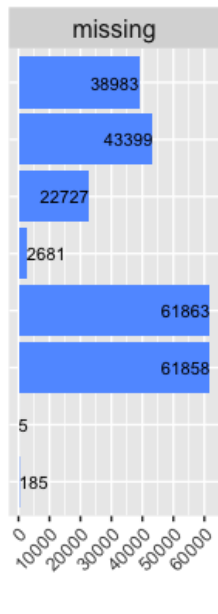

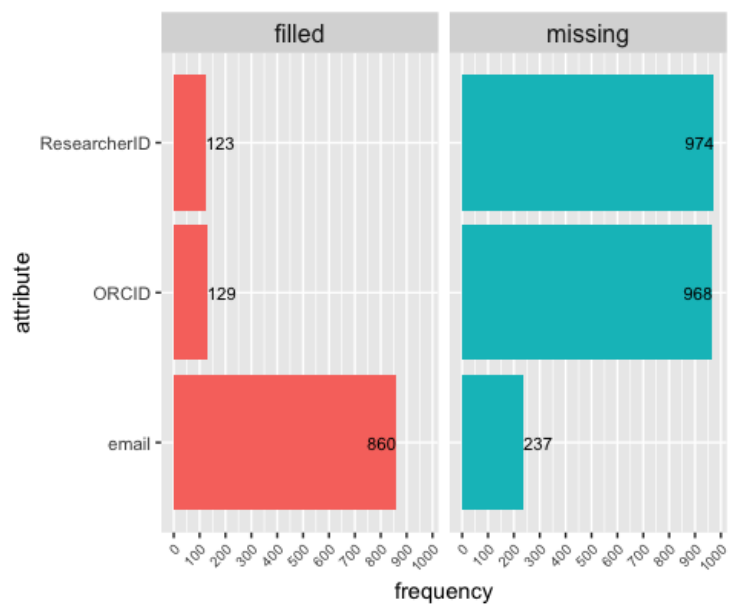

Figure 1. Missing values for some paper markers (left) and personal attributes (right)

\section{Methods and Tools}

The implemented solution tries to identify the UAIC authors of Clarivate papers in three steps. Firstly, highly plausible and highly implausible authors are identified based on the corroboration of paper's EM, OI, RI and C1 markers (see section 2) with UAIC members' declared e-mail addresses, ORCID, ResearcherID, and aliases. In the second step, titles are extracted for both plausible and implausible authorships. After tokenization and lemmatization [5], stop words are removed. Table frequencies and other metrics are computed for title unigrams and bigrams for each UAIC member, domain of research and UAIC department. In step 3 the metrics are employed for building predictive models for author identification of the papers lacking authors e-mails, ORCID and ResearcherID. Classification models were built with random forests due to their good performances [6].

Data cleaning, visualization and analysis were performed in R [7]. The 'tidyverse` ecosystem of packages [8] was the main tool for data processing operations. Levenstein distance and JaroWinkler distance were computed with 'stringdist' package [9]. Text mining operations (tokenization, lemmatization) were performed with package 'tidytext' [5]. Classification models were built, tuned and assessed with packages `ranger` [10] and 'caret' [11].

\section{Results and Discussion}

From Clarivate (ISI) Web of Science platform (https://clarivate.com) papers were imported by filtering them on affiliation (UAIC) and UAIC members' names and aliases. Selection on aliases was important since not all of the papers contain authors affiliation, or sometimes the affiliation refers not to UAIC, but to a faculty, department or lab within UAIC. Importing papers by person aliases have the major advantage of extracting works published under a previous name (maid family name, pre-divorce name, etc.) but also introduces some noise. For example, papers published by UAIC member 'Georgescu Mircea Radu' presents in the Clarivate database the following variants: 'Georgescu, Mircea Radu'; 'Georgescu, MR'; 'Georgescu, Mircea', 'Georgescu, M'. In this case, the second surname (Radu) is not used alone, but only in combination with the first (Mircea). Other authors preferred to use only the second surname in their papers. Consequently, when extracting papers for authorship identification, the threshold for the Levenstein distance between paper's author and the person aliases ( $\left.l v \_d i s t \_a u t h o r \_a l i a s\right)$ was set to 1 . When multiple aliases share the same Levenstein distance to the paper's author name, the alias with the least Jaro-Winkler distance was kept [12]. Implausible authors were determined when papers had e-mail addresses, or ORCIDs, or 
ResearcherIDs for all of its authors and no UAIC member (with an alias close to one of the authors) has such an address/id.

For every extracted paper, the title was lemmatized and tokenized, stop words were removed, and then frequency tables were determined for unigrams and bi-grams [5].

Also, for the highly plausible authors identified by matching of paper's EM, OI, RI and C1 markers with UAIC members' declared e-mail addresses, ORCID, ResearcherID, and aliases, all the paper titles were extracted, lemmatized and tokenized. The author's profile was the result of processing both plausible and implausible papers with metrics associated on groups of persons and unigrams/bigrams of their papers title:

- points_of_this_word_for_the_person: considers the frequency of word and the length of the word, assigning greater weight to bigrams (relative to unigrams)

- points_of_this_unique_word_for_the_person: formula is similar to the previous metric, but points are computed only for the words that appear exclusively in the current person's titles and not in other UAIC members' works.

- points_of_this_word_for_the_person_implausible: similar to points_of_this_word_ for_the_person metric, considering only the papers with authors whose names are close to the current person, but they are almost certain to be authored by other people (because even if their name is similar, the article e-mails, ORCIDs, ResearcherIDs and affiliations do not contain any information pointing to the current person).

- ratio_points_of_this_word_for_the_person adjusts the points_of_this_word_for_ the_person with the sum of the points for all identified words of the current person.

- ratio_points_of_this_unique_word_for_the_person: same as ratio_points_of_this_ word_for_the_person, but it is computed only for the words appearing exclusively in current person's papers titles.

- ratio_points_of_this_word_for_the_person_implausible: computed similar to ratio_ points_of_this_word_for_the_person, but considers only implausible papers of the current person.

A set of similar metrics were computed on the domains of research reported by the UAIC member (also available in UAIC database). The domain profile is then built by aggregating all the points and ratios for UAIC authors affiliated to this domain. The idea targets new faculty members and also authors whose recent papers approach new topics that already have been covered by some domain fellow. Individual metrics were also aggregated on departments. Both domain and department aggregates were considered because many UAIC departments cover multiple domains of study and research. Individual, domain and departmental metrics were joined with paper metric by their common/matching words (unigrams and bigrams). After grouping on paper id (wos code) and person id, the final dataframe contained 23 predictors, such as:

- sum_points_match_paper: sum of the points for common words of the current paper and current person at the paper level;

- sum_ratios_match_paper: adjust sum_points_match_paper relative to all the words contained in the title of the current paper;

- sum_points_match_person: total points of all common words (paper-person) at the person level;

- sum_points_match_unique_person: total points of all common words (paper-person) at the person level, computed only for unigrams and bigrams which are unique (in UAIC) to the current person; 
- sum_points_unmatch_person: total points of all common words (paper-person) at the person level, but extracted only from the papers where current person is not supposed to be the (co)author;

- sum_ratios_match_person: adjust sum_points_match_person with the total number of points for all one- and bi-gram words of the person's papers titles;

- sum_ratios_match_unique_person: similar to sum_ratios_match_person, but takes into consideration only words appearing exclusively (relative to UAIC) in current person's papers titles;

- sum_ratios_match_unique_person: similar to sum_ratios_match_person, but takes into consideration only words appearing exclusively (relative to UAIC) in current person's papers titles;

- sum_ratios_unmatch_person: similar to sum_ratios_match_person, but takes into consideration only the title one- and bi-gram tokens from the implausible papers of the current person.

Predictors computed at domain and department level share the name same as above, but the ¿_person` suffix is replaced with `_domain` and __dept` respectively.

The outcome variable of the random forest models was is_this_the_author. To avoid overfitting, from the training dataset all the observations related to highly plausible and highly implausible authorship were removed. Instead they were included in the validation set.

The Random Forest model was tuned by a grid search, varying successively the hyperparameter $m$ try from 2 to 10 and keeping the parameter number of trees to value 500 (No noticeable improvement was recorded when setting larger number of trees). Minimum prediction error was recorded for mtry $=6$. Consequently, the final RF model was build based on this value of the hyperparameter. In assessing model performance on the validation set, predicted values were compared against the papers UAIC members confirmed (or infirmed) to be the (co)authors. Estimated model Accuracy 0.91 (95\% CI : [0.90, 0.91] which is good relative the No Information Rate of 0.80 . Sensitivity was estimated at 0.93 and Specificity at 0.90 . Reported Area Under Curve (figure 2-left) was $96.49 \%$.
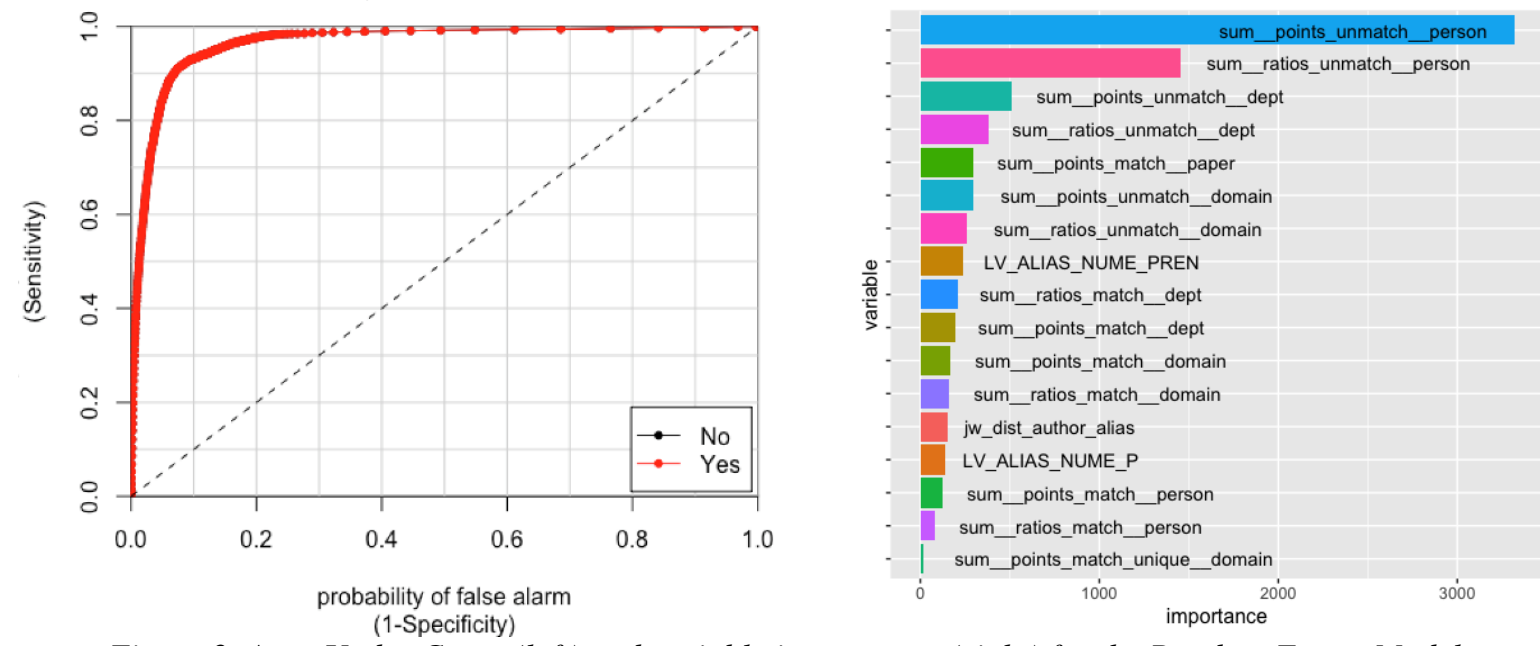

Figure 2. Area Under Curve (left) and variable importance (right) for the Random Forest Model

The right side of figure 2 shows the model variable importance. Most relevant predictors are related to the papers whose UAIC (co)authorship is implausible. This might seem intriguing when comparing with Zantout et. al. [2] findings. In our model, good predictions are related not only to matching, but also to the un-matching of paper's title and the person's (papers) titles. 
www.conferenceie.ase.ro

\section{Conclusion}

Based on Clarivate metadata and basic personal information about UAIC members, the solution presented in this paper estimates the plausibility of authorship for UAIC members on Clarivateindexed works using a predictive model built with random forests.

The model displays good performance. Also, the solution does not require the full text of the paper (which could trigger copyright infringements), but only the paper title and some other metadata.

Further research must address the problem of model tuning or even re-examination of the predictors. Also results might be corroborated with other classification techniques results (e.g. CART, boosting), and also other text analysis methods, such as topic modeling (Latent Dirichelet Analysis), stylometry, etc.

\section{Acknowledgment}

Al. I. Cuza University of Iasi, GRANT 12521: "Platforma de servicii software privind centralizarea raportarii activitatii stiintifice la nivel UAIC, prin integrarea datelor referitoare la publicatii si proiecte de cercetare".

\section{References}

[1] A. A. Ferreira, M. A. Gonzcalves, and A. H. Laender, "A Brief Survey of Automatic Methods for Author Name Disambiguation", SIGMOD Record, vol. 41, no. 2, pp. 15-26, 2012

[2] R. Zantout, Z. Osman, and L. Hamandi, "A Universal Method for Author Identification Using Statistical Properties of Text," in Proc. of the 2nd International Conference on Vision, Image and Signal Processing (ICVISP 2018). ACM, New York, 2018, Article 20

[3] T. Neal, K. Sundararajan, A. Fatima, Y. Yan, Y. Xiang, and D. Woodard, "Surveying Stylometry Techniques and Applications", ACM Comput. Surv., vol. 50, no. 6, Article 86 (November 2017)

[4] M. Rosen-Zvi, C. Chemudugunta, T. Griffiths, P. Smyth, and M. Steyvers, "Learning authortopic models from text corpora", ACM Trans. Inf. Syst. 28, 1, Article 4 (January 2010)

[5] J. Silge and D. Robinson, Text Mining with R. A Tidy Approach. Sebastopol, CA: O'Reilly, 2017.

[6] T. Hastie, R. Tibshirani, J. Friedman, The Elements of Statistical Learning: Data Mining, Inference, and Prediction (2nd ed.). New York: Springer, 2009

[7] R Core Team. 2019. R: A Language and Environment for Statistical Computing. Vienna, Austria: R Foundation for Statistical Computing. https://www.R-project.org/ [Jan. 22, 2019]

[8] H. Wickham and G, Grolemund, R for Data Science: Import, Tidy, Transform, Visualize, and Model Data. Sebastopol, CA: O'Reilly, 2017.

[9] M. van der Loo, "The stringdist package for approximate string matching", The R Journal, vol. 6, pp. 111-122. <URL: https://CRAN.R-project.org/package=stringdist>.

[10] M.N. Wright, A. Ziegler (2017). "ranger: A Fast Implementation of Random Forests for High Dimensional Data in C++ and R", Journal of Statistical Software, vol. 77 no. 1, pp.1-17.

[11] M. Kuhn et al. (2018). "caret: Classification and Regression Training. R package version 6.081", https://CRAN.R-project.org/package=caret

[12] W.W. Cohen, P. Ravikumar, and S. E. Fienberg, "A comparison of string distance metrics for name-matching tasks," in Proc of the 2003 International Conference on Information Integration on the Web (IIWEB'03), Subbarao Kambhampati and Craig A. Knoblock (Eds.). AAAI Press, 2003, pp.73-78. 